\title{
Echocardiographic evaluation of diastolic functions in patients with polycystic ovary syndrome: A comperative study of diastolic functions in sub-phenotypes of polycystic ovary syndrome
}

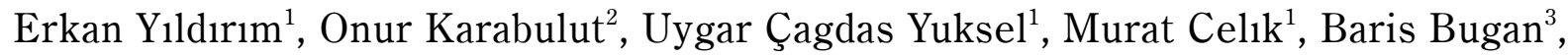 \\ Yalcin Gokoglan ${ }^{1}$, Mustafa Ulubay ${ }^{4}$, Mutlu Gungor ${ }^{5}$, Mustafa Koklu ${ }^{1}$ \\ ${ }^{1}$ Department of Cardiology, Gulhane Research and Training Hospital, Ankara, Turkey \\ ${ }^{2}$ Department of Obstetrics and Gynecology, Ataturk Research and Training Hospital, Ankara, Turkey \\ ${ }^{3}$ Department of Cardiology, Corlu State Hospital, Tekirdag, Turkey \\ ${ }^{4}$ Department of Obstetrics and Gynecology, Gulhane Research and Training Hospital, Ankara, Turkey \\ ${ }^{5}$ Department of Cardiology, Memorial Sisli Hospital, Istanbul, Turkey
}

Background: Polycystic ovary syndrome (PCOS) is a heterogeneous endocrine disorder among reproductive-aged women. It is known to be associated with cardiovascular diseases. The aim of this study was to determine and compare the echocardiographic data of patients according to the phenotypes of PCOS.

Methods: This study included 113 patients with PCOS and 52 controls. Patients were classified into four potential PCOS phenotypes. Laboratory analyses and echocardiographic measurements were performed. Left ventricular mass was calculated by using Devereux formula and was indexed to body surface area.

Results: Phenotype-1 PCOS patients had significantly higher homeostasis model assessment - insulin resistance $(H O M A-I R)(p=0.023)$, free testosterone $(p<0.001)$, LDL cholesterol levels $(p<0.001)$ and free androgen index $(p<0.001)$ compared with the control group. There were significant differences between groups regarding the septal thickness, posterior wall thickness, Left ventricular ejection fraction, E/A ratio and left ventricular mass index (for all, $p<0.05$ ). PCOS patients with phenotype 1 and 2 had significantly higher left ventricular mass index than the control group $(p<0.001)$. In univariate and multivariate analyses, PCOS phenotype, modified Ferriman-Gallwey Score and estradiol were found as variables, which independently could affect the left ventricular mass index.

Conclusions: This study showed that women in their twenties who specifically fulfilled criteria for PCOS phenotype-1 according to the Rotterdam criteria, had higher left ventricular mass index and decreased E/A ratio, which might be suggestive of early stage diastolic dysfunction. (Cariol J 2017; 24, 4: 364-373)

Key words: polycystic ovary syndrome, echocardiography, diastolic dysfunction, left ventricular mass

\section{Introduction}

Polycystic ovary syndrome (PCOS) is a heterogeneous endocrine disorder among reproductive- aged women with an estimated prevalence of $5 \%$ to $10 \%$ [1]. Despite the ongoing debate on the diagnostic criteria, there is a wide consensus that the PCOS is diagnosed when two of the three fol-

Address for correspondence: Erkan Yildırım, MD, Department of Cardiology, Gulhane Research and Training Hospital, 06018 Etlik-Ankara, Turkey, tel: +90 53076163 98, e-mail: dr_erkanyildirim@yahoo.com.tr 
lowing features are present; irregular menstrual cycles due to oligo- or anovulation, evidence of biochemical or clinical androgen excess and polycystic ovaries [2]. Introduction of these broadened criteria resulted in a considerable increase in the prevalence of PCOS [3].

PCOS is known to be associated with a cluster of adverse cardiovascular (CV) features such as obesity, insulin resistance, hypertension and consequently an increased risk of $\mathrm{CV}$ disease (CVD) $[4,5]$. But due to heterogeneous clinical expression of PCOS, data on the association of PCOS with CVD have been inconsistent. Better definition of sub-phenotypes related with increased CV risk is required. Currently there are no consensus guidelines regarding screening for CVD in patients with PCOS. Considering that risk is not same in all PCOS patients, it remains to be elucidated to what extent women presenting with PCOS face increased long-term CV risk. Previous studies have not assessed whether the overall PCOS phenotype or the individual components of PCOS are associated with the development of subclinical CVD. Identification of the early asymptomatic impairment of left ventricular (LV) function may be an important indicator in preventing a progression to overt heart disease.

The aim of this study was to determine and compare the echocardiographic data of patients among the sub-phenotypes of PCOS with those of healthy subjects by using conventional echocardiographic methods and tissue Doppler imaging. Thus, the aim was to identify which PCOS sub-groups are strongly associated with increased CV risk.

\section{Methods}

\section{Patient population}

This cross-sectional observational study was conducted by Cardiology and Obstetrics and Gynecology Department of our institute. A total of 113 patients with PCOS and 52 controls were enrolled in this study. Study groups were matched with respect to age and body mass index (BMI). Subjects were informed regarding the purpose of the study and provided written informed consent. Local Ethics Committee of our institute approved the protocol of the study.

\section{Definitions}

PCOS was diagnosed according to the Rotterdam criteria in the presence of at least two of the following three features: oligo- or anovulation (ANOV), clinical and/or biochemical hyperandro- genism (HA), and polycystic ovarian morphology in one or both ovaries (PCOM) [2]. Afterwards, women diagnosed with PCOS were classified into one of four potential PCOS phenotypes:

- phenotype 1: HA + ANOV + PCOM;

- phenotype 2: HA + ANOV;

- phenotype 3: HA + PCOM;

- phenotype 4: ANOV + PCOM.

The following exclusion criteria were used in the selection of patients and controls: any CV disorder (including hypertension), diabetes mellitus, thyroid and renal diseases, pregnancy or breastfeeding, hyperprolactinaemia, Cushing's syndrome, congenital adrenal hyperplasia, history of any neoplastic disorder, chronic alcohol consumption and current or previous use of glucocorticoids, oral contraceptives, ovulation induction agents, antiandrogens, other hormones, anti-hypertensive, anti-diabetic drugs. Women who could not be assigned to a specific PCOS phenotype were also excluded from the study. Control subjects were chosen among healthy women with no clinical or biochemical evidence of hyperandrogenaemia or hirsutism, regular menstrual cycles and normal ovaries on ultrasonography scan.

The screening began with a thorough detailed history inquiry. Subsequently, anthropometric measurements were performed, including height, weight, BMI, systolic and diastolic blood pressure (SBP, DBP), waist circumference (measured midway between the arcus costae and anterior superior iliac spine), hip circumference (measured at the level of the anterior superior iliac spine), and the measurement of hirsutism with the use of the modified Ferriman-Gallwey Score (m-FGS). The waist-to-hip ratio (WHR) was also calculated. Arterial blood pressure was measured using a mercury sphygmomanometer; the mean of three measurements of SBP and DBP was taken while the subjects were sitting after a 10-min rest. BMI was calculated as weight in kilograms divided by the square of height in meters $\left(\mathrm{kg} / \mathrm{m}^{2}\right)$. Metabolic syndrome was defined as having at least three of the following three features: increased waist circumference $(>88 \mathrm{~cm}$ ), increased blood pressure (SBP $\geq 135 \mathrm{~mm} \mathrm{Hg}$, DBP mm Hg $\geq 85$, or treatment for hypertension), increased fasting glucose $(\geq 100 \mathrm{mg} / \mathrm{dL}$ or taking medications for diabetes), increased triglycerides $(\geq 150 \mathrm{mg} / \mathrm{dL})$, and decreased high density lipoprotein cholesterol ( $<40 \mathrm{mg} / \mathrm{dL}$ ). Oligo-anovulation was defined as oligomenorrhea (mean bleeding interval 35-182 days in last six menstrual bleeds) or amenorrhea (absence of menstrual bleeding for $>182$ days). Clinical and biochemical hyperandrogenism were 
defined as $\mathrm{m}-\mathrm{FGS} \geq 8$, and/or a free androgen index (FAI: [Total testosterone $(\mathrm{nmol} / \mathrm{L}) / \mathrm{sex}$ hormone-binding globulin $(\mathrm{nmol} / \mathrm{L}) \times 100])>4.5[6]$. PCOM was defined as $\geq 12$ follicles measuring $2-9 \mathrm{~mm}$ in diameter in at least one ovary, or increased ovarian volume (>10 mL). Baseline venous blood was sampled in the morning after an overnight fast. The following endocrinologic and metabolic laboratory parameters were selected for comparative analysis between PCOS phenotypes and the control groups: follicle stimulating hormone ( $\mathrm{FSH})$, luteinizing hormone (LH), estradiol (E2), free testosterone, total testosterone, dehydroepiandrosterone sulfate (DHEA S), sex hormone-binding globulin (SHBG), prolactin, insulin, glucose, total cholesterol (TC), triglycerides (TG), and high-density lipoprotein cholesterol (HDL-C), low-density lipoprotein cholesterol (LDL-C). Insulin resistance was assessed with the use of the homeostasis model assessment (HOMA-IR: [fasting glucose $(\mathrm{mg} / \mathrm{dL}) \times$ fasting insulin $(\mu \mathrm{IU} / \mathrm{mL})] / 405)$ [7]. The cut-off value for HOMA-IR was taken as 2.7.

\section{Echocardiographic evaluation}

After a 15-min resting period, the patients were examined in the left lateral decubitus position with a Vivid S5 (GE Healthcare, Norway). Complete 2D, color, pulsed and continuous-wave Doppler examination were performed according to the standard techniques. Measurements of $\mathrm{LV}$, left atrial dimension and wall thickness were performed according to the recommendations of the American Society of Echocardiography (ASE) guidelines using parasternal long- and short-axis views [8]. LV mass (LVM) was calculated from 2 -dimensional echocardiographic measurements by using Devereux formula: $\mathrm{LVM}=1.04 \times$ [(IVST + PWT + LVDd $\left.)-(\text { LVDd })^{3}\right]-13.6$ and was indexed to body surface area [9]. Left ventricular hypertrophy (LVH) was defined as a LVM index (LVMI) greater than $95 \mathrm{~g} / \mathrm{m}^{2}$, which is an upper limit of normal LVM in women. Categorizations of LVH either concentric or eccentric hypertrophy were done according to recommendations of the ASE guidelines [8]. A modified Simpson's biplane method was applied to calculate LV ejection fraction (LVEF). Trans-mitral pulsed-wave Doppler velocity were recorded from the apical four-chamber view with Doppler sample placed between the tips of the mitral leaflets. Early (E) and late (A) diastolic wave velocity, $\mathrm{E} / \mathrm{A}$ ratio were measured from the mitral inflow profile. The mitral annular myocardial velocity was obtained from the two- and four-chamber view and tricuspid annular myocardial velocity was obtained from the four-chamber view by placing a tissue Doppler sample volume. The Nyquist limit was adjusted to $15-20 \mathrm{~cm} / \mathrm{s}$ with minimum gain and low-filter settings. Early (Em) and late (Am) diastolic velocity was obtained. Pulsed-wave tissue Doppler recordings of the lateral portion of the mitral annulus were used for the assessment of peak early diastolic mitral annular velocity $(\mathrm{Em})$. The ratio of the mitral inflow early diastolic velocity to the peak early diastolic annular velocity (E/ $\mathrm{Em})$ was computed to estimate LV filling pressure.

\section{Statistical analyses}

Baseline variables were expressed as means with standard deviation or numbers with percentage. Multiple imputation and other statistical analyses were performed with the use of SPSS statistics version 20.0. Group comparisons were performed by one-way ANOVA with Tukey's post hoc test. To determine the independent variables likely to affect the LVMI, a multivariate linear regression analysis was performed with a backward selection process including parameters, which were clinically important, found significant in univariate correlation analysis and significantly differed between the groups. Differences were considered statistically significant when the $\mathrm{p}$ value was $<0.05$.

\section{Results}

\section{Demographic and clinical results}

Clinical characteristics and laboratory findings of patients and the control group are shown in Tables 1 and 2. PCOS population involved $41(36 \%)$ patients with phenotype-1 (ANOV + HA + PCO), 20 (17\%) patients with phenotype-2 (ANOV + HA), 25 (22\%) patients with phenotype-3 (HA + PCO) and 27 patients (23\%) with phenotype-4 (ANOV + PCO). The four-phenotype groups and the control group were similar in terms of fasting glucose, fasting insulin, and presence of metabolic syndrome, FSH, estradiol, prolactin, triglycerides, total testosterone, androstenedion, and DHEAS. There were differences between groups regarding age, SBP and DBP, BMI, WHR, m-FGS, HOMA-IR, LDL levels, LH/FSH ratio, free testosterone, SHBG and FAI. Compared with the control group all four phenotypes of PCOS were noted to have higher SBP and DBP $(\mathrm{p}<0.001)$. Subjects from both phenotype- 1 and phenotype-3 PCOS had higher BMI than the control group $(\mathrm{p}=0.020)$. Phenotype-1 PCOS patients had significantly higher HOMA-IR $(2.66 \pm 1.61$ vs. $1.91 \pm 0.89$; $\mathrm{p}=0.023)$, free testosterone $(2.75 \pm 1.40 \mathrm{vs} .1 .68$ 


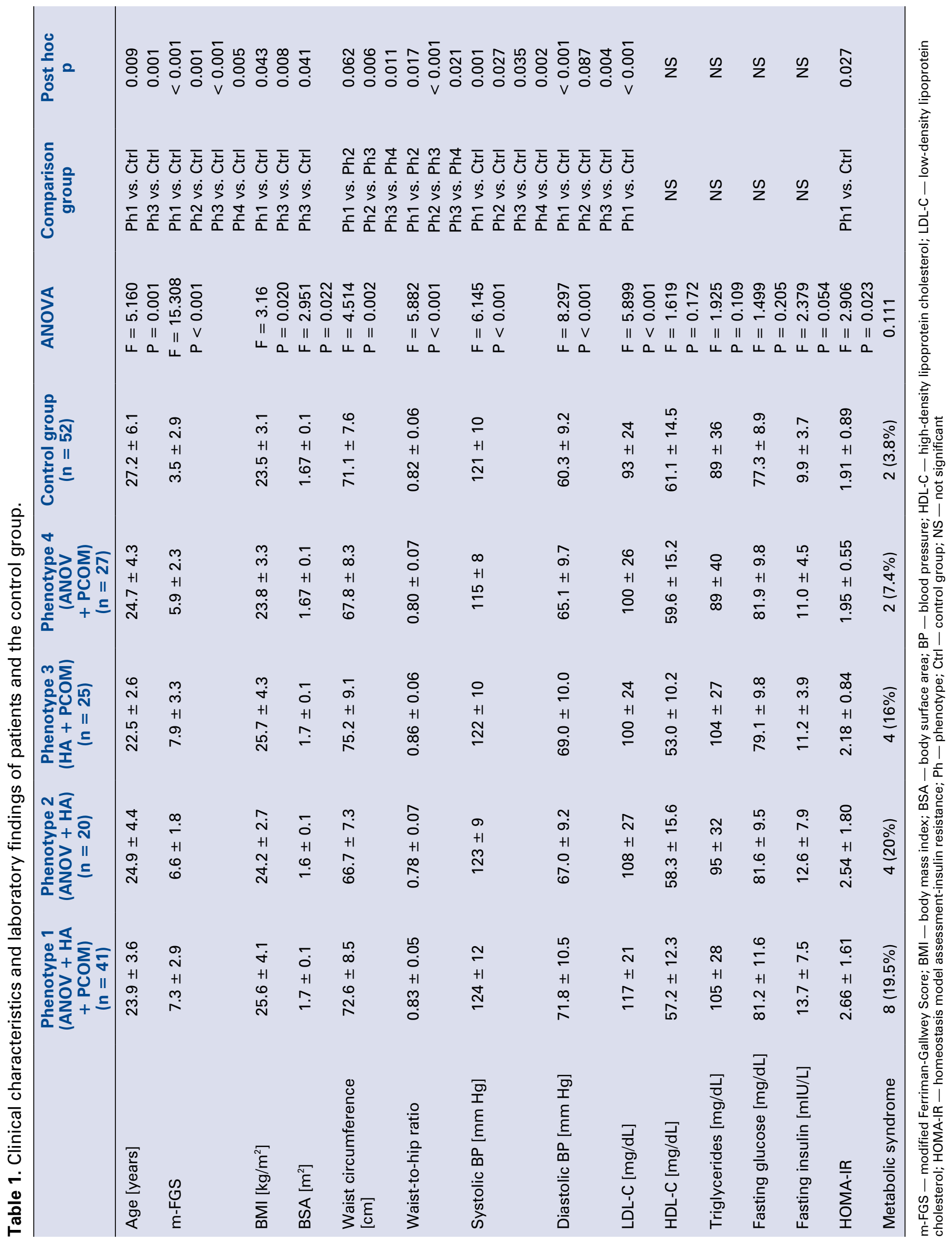




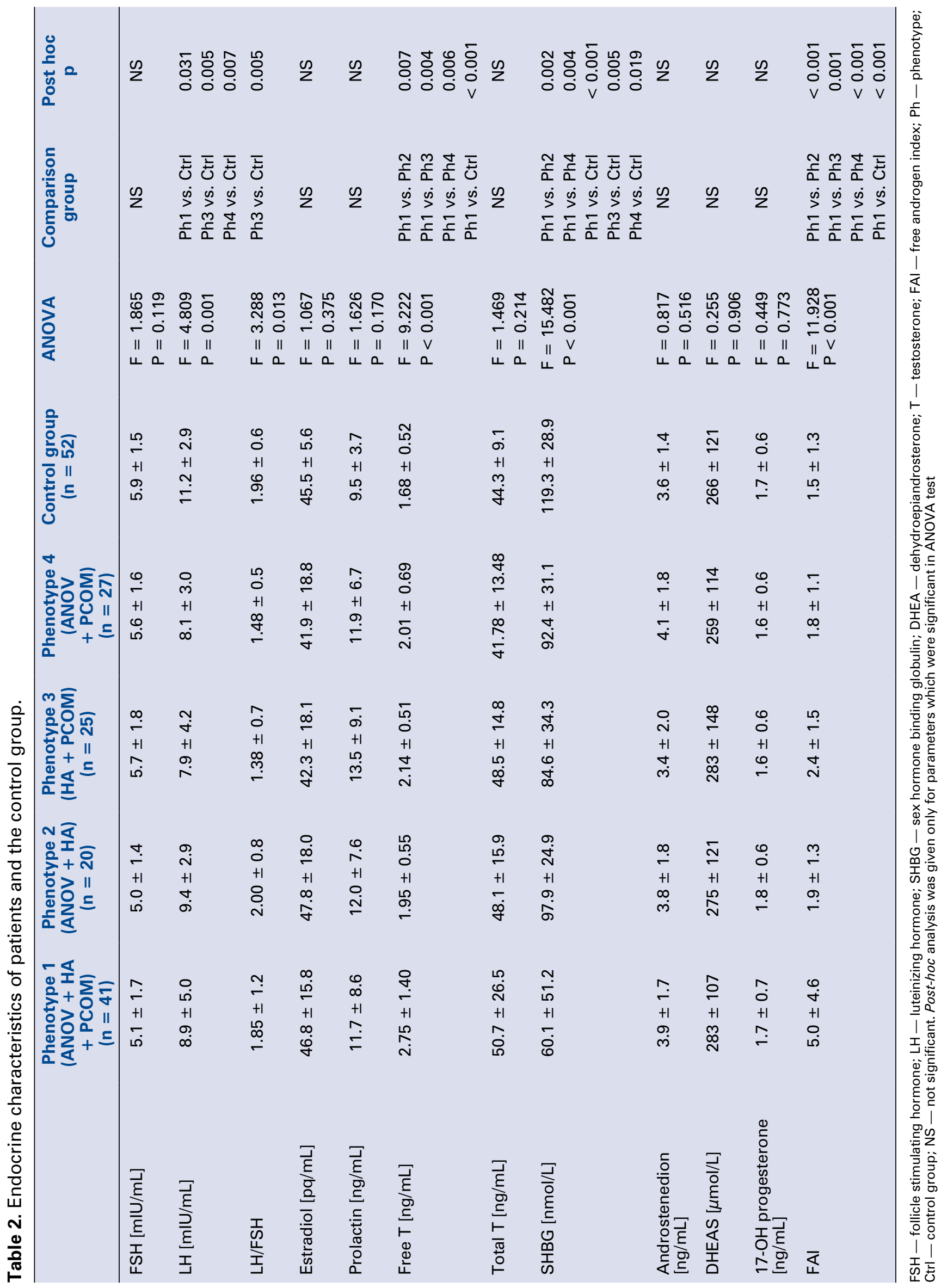




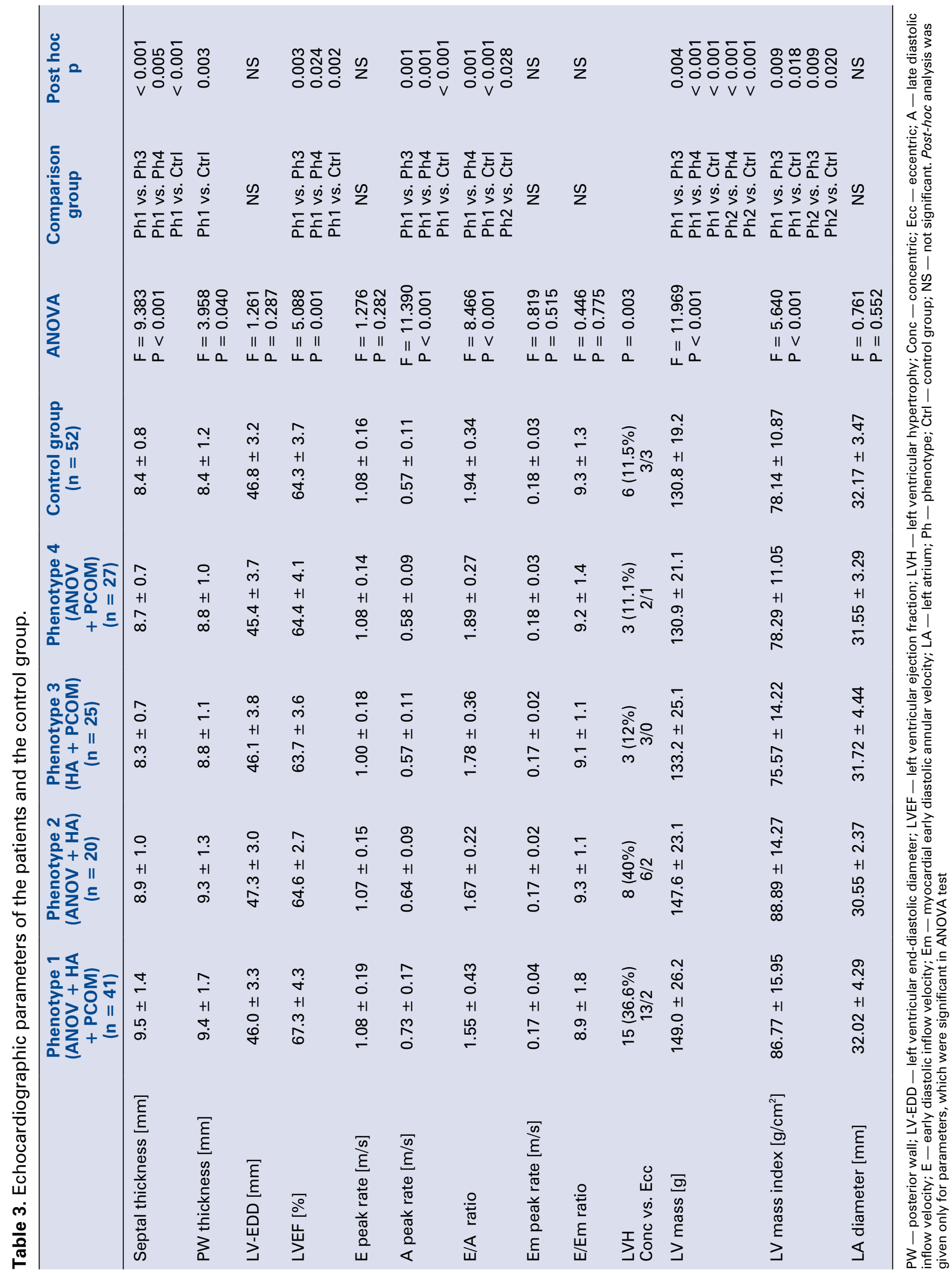


Table 4. Univariate and multivariate analysis to determine the variables likely to affect the left ventricular mass index.

\begin{tabular}{|c|c|c|c|c|c|}
\hline \multirow[t]{2}{*}{ Variable } & \multicolumn{2}{|c|}{ Univariate analysis } & \multicolumn{3}{|c|}{ Multivariate analysis } \\
\hline & $\mathbf{r}$ & $\mathbf{p}$ & $\begin{array}{l}\text { Unstandardized } \\
\text { Coefficient } \beta\end{array}$ & $95 \% \mathrm{Cl}$ & p \\
\hline PCOS phenotype & -0.277 & $<0.001$ & -4.221 & $-5.569-2.874$ & $<0.001$ \\
\hline m-FGS & -0.164 & 0.035 & -1.527 & $-2.177--0.877$ & $<0.001$ \\
\hline E2 & -0.166 & 0.033 & -0.148 & $-0.280--0.015$ & 0.029 \\
\hline LDL-C & 0.190 & 0.014 & 0.060 & $-0.019-0.139$ & 0.137 \\
\hline
\end{tabular}

$\mathrm{Cl}$ - confidence interval; PCOS - polycystic ovary syndrome; m-FGS - modified Ferriman-Gallwey Score; E2 - estradiol; LDL-C — lowdensity lipoprotein cholesterol. Parameters included in the multivariate analysis were: age, free androgen index (FAI), systolic blood pressure presence of metabolic syndrome, PCOS phenotype, m-FGS, E2, homeostasis model assessment-insulin resistance (HOMA-IR) and LDL-C

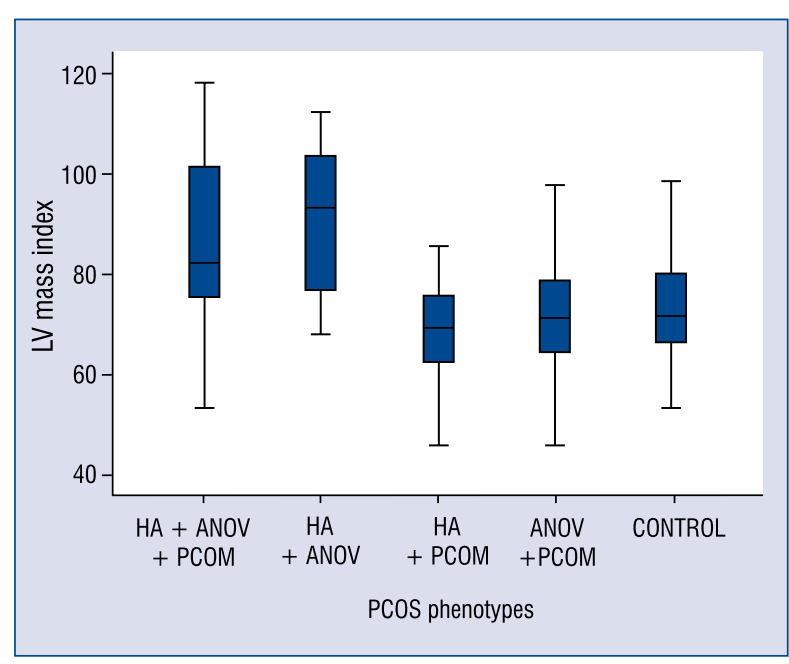

Figure 1. Left ventricular (LV) mass index of four phenotypes and the control group; ANOV - oligo- or anovulation; HA - hyperandrogenism; PCOM - polycystic ovarian morphology; PCOS - polycystic ovary syndrome.

$\pm 0.52 ; \mathrm{p}<0.001)$, LDL levels $(117.14 \pm 21.27 \mathrm{vs}$. $93.51 \pm 24.06 ; \mathrm{p}<0.001)$ and FAI $(5.08 \pm 4.69$ vs. $1.57 \pm 1.38 ; \mathrm{p}<0.001)$ compared with the control group whereas other phenotypes and control group were similar.

\section{Echocardiographic results}

The echocardiographic data of patients and the control group included in the study are shown in Table 3. The four phenotypes and the control group did not differ regarding LV dimensions, end--diastolic volume, end-systolic volume, E peak rate, Em peak rate and $\mathrm{E} / \mathrm{Em}$ ratio. But there were significant differences between groups regarding the septal thickness, posterior wall thickness, LVEF, E/A ratio and LVMI. Although the E/A ra- tio was significantly decreased in all phenotypes compared to the control group, this decrease was prominent in phenotype-1 PCOS. Regarding the LVEF, subjects with phenotype-1 PCOS differed significantly compared to the control and phenotypes $2-4$ groups $(p=0.001)$. Significant differences were also found between PCOS patients and control group regarding the presence of $\mathrm{LVH}$ $(p=0.003)$. Subjects with phenotype 1 and 2 PCOS had significantly higher LVMI values than the control group $(\mathrm{p}<0.001)$, while subjects with phenotype 3 and 4 did not have a higher LVMI compared with the control group (Fig. 1).

All parameters evaluated in this study were entered for univariate analysis, and only parameters correlated significantly with LVMI were specified in the table. In univariate correlation analysis, PCOS phenotype, m-FGS, E2 and LDL level correlated significantly with LVMI ( $p<0.05$, for all) (Table 4). Subsequently, a backward multivariate linear regression analysis was performed to determine the independent variables likely to affect the LVMI, which were clinically important, found significant in univariate correlation analysis and significantly differed between groups. Variables entered into the multivariate linear regression analysis were age, FAI, SBP, presence of metabolic syndrome, PCOS phenotype, m-FGS, estradiol (E2), HOMA-IR and LDL-C. Multivariate analysis showed that phenotype $(\mathrm{p}<0.001), \mathrm{m}-\mathrm{FGS}$ $(\mathrm{p}<0.001)$, and E2 $(\mathrm{p}=0.029)$ were independently associated with LVMI (Table 4).

\section{Discussion}

This study demonstrated that the presence of PCOS phenotype- 1 and -2 in young women, is clearly associated with an increased LVMI and decreased $\mathrm{E} / \mathrm{A}$ ratio compared to the control group. 
These echocardiographic findings might indicate the early stage of cardiac diastolic dysfunction and/or abnormalities in the filling phase of the left ventricle.

It is a established fact that diastolic dysfunction might be one of the first echocardiographic abnormalities in patients with hypertension and atherosclerotic heart disease [10]. There is compelling evidence that patients with PCOS have increased $\mathrm{CV}$ risk compared with age-matched controls. Thus, this association was not surprising because of the higher incidence of diabetes mellitus, obesity, impaired glucose tolerance and metabolic syndrome in this patient population [11]. Because CV involvement was seen subsequently with PCOS, ventricular diastolic dysfunction may be an early indicator of impending heart disease.

A group of PCOS related factors could potentially contribute to LV dysfunction in PCOS including hormonal aberrations, obesity, endothelial dysfunction and insulin resistance (IR) [12]. Obesity has been estimated to affect more than $50 \%$ of women with PCOS [13]. The pathophysiologic contribution of obesity to LV dysfunction includes chronic overload with LVH increased oxygen consumption, lipotoxicity associated cardiac steatosis, myocardial fibrosis and overproduction of cardio-inhibitory cytokines [14]. Although our patient population and control group subjects were not obese, BMI was significantly higher in phenotype-1 group. This finding supports that being overweight may be one of the contributors of impaired LV relaxation in patients with PCOS.

Insulin resistance is a common abnormality in patients with PCOS [15]. But approximately 50\% of obese women with PCOS may not have documented IR by intensive testing. [13]. IR may lead to increased sodium retention with subsequent blood volume increase, myocyte growth and fibrosis and activation of the sympathetic nervous system that causes unfavorable effects on CV system $[14,16]$. An association was also demonstrated between IR and coexisting vascular endothelial dysfunction and impaired mitochondrial oxidative metabolism that may impair the myocardial functions [17]. In this study by Victor et al. [17], authors concluded that the inflammatory state that affects the endothelial function is related to IR in PCOS patients. The present findings showed that only phenotype- 1 PCOS group had significantly higher HOMA-IR compared to control subjects. This finding is consistent with the previous reports that show relationship between LV diastolic dysfunction and IR $[14,16,18]$.
In this study, phenotype-1, which can be defined as a severe form of PCOS was significantly differed from the control group regarding the variables such as LVMI, HOMA-IR, FAI, free testosterone and LDL levels. Higher FAI and free testosterone levels in this group were consistent with hyperandrogenism. Evidence from recent studies have demonstrated that hyperandrogenic PCOS phenotype encompasses the patients with the most unfavorable cardiometabolic profiles $[19,20]$. Therefore, this study is consistent with previous reports and hyperandrogenism might be one of the contributors of impaired LV diastolic function in PCOS patients.

Previous studies investigating PCOS and conventional echocardiographic parameters are limited to small case control studies and the results are not consistent due to underlying differences in defining the PCOS population [21-25]. Some previous studies reported no significant differences in certain echocardiographic measures of cardiac function between PCOS patients and healthy subjects including ejection fraction, mitral E/A ratio, and tissue Doppler parameters [22, 25]. In a study, Tiras et al. [24] reported evidence of diastolic dysfunction in patients with PCOS based on flow parameters such as isovolumetric relaxation time (IVRT), atrial filling time, and pre-ejection time. However, they found no differences in E/A ratio, left atrium diameter index or LVMI [24]. Orio et al. [21] also reported a $40 \%$ higher mean LVMI in PCOS women compared with age and BMI matched controls.

Increased LVMI usually occurs as a result of stressors such as hypertension or increased wall stress in LV cavity. It is often an early sign of diastolic dysfunction, or failure of the heart to relax and fill during diastole [26]. In this study, it was found that mean E/A values and LVM indices of each group reside within the normal ranges as depicted in Table 3. However when compared with the healthy controls significant decrease in E/A ratio and increase in LVMI was observed in phenotype 1 and 2. Besides LVH was more prevalent in phenotype 1 and 2. Despite the normal mean values, this finding suggests cardiac remodeling is taking place in early years of life in those phenotypes and may lead to overt clinical cardiac dysfunction in the feature. In this study PCOS phenotype was found as an independent predictor of LVMI. PCOS is related with increased blood pressure but LV structural changes in PCOS is shown to be independent from blood pressure changes [21, 27]. In the present study, blood pressure levels were com- 
parable among each PCOS phenotype and healthy controls. What causes LV structural changes in PCOS remains to be elucidated. These results are consistent with the previous reports showing higher risk for CVD in PCOS subjects $[28,29]$. The $\mathrm{E} / \mathrm{A}$ ratio is also one of the most widely used parameters for determining diastolic dysfunction. Reduced $\mathrm{E}$ wave velocity and increased $\mathrm{A}$ wave velocity (or a decreased $\mathrm{E} / \mathrm{A}$ ratio) is suggestive of impaired relaxation pattern related with diastolic dysfunction. Consistent with our results Yarali et al. [30] reported that PCOS patients had decreased mitral E/A ratio and shorter IVRT compared to control group. Nevertheless, there are some other studies, which reported no differences in mitral $\mathrm{E}, \mathrm{A}$ velocities and $\mathrm{E} / \mathrm{A}$ ratio between the PCOS patients and controls [25].

Considering that risk is not the same in all patients, it remains to be elucidated that the extent to which hormonal and metabolic abnormalities of PCOS might contribute to cardiac dysfunction and which phenotype of women presenting with PCOS face increased long-term CV risk. In comparison with previous studies, this study compares PCOS patients with different phenotypes, as well as its individual components such as HA, ANOV and PCOM. The present study demonstrated that women especially, who fulfilled criteria for PCOS phenotype-1 showed evidence for both higher LVMI and decreased $\mathrm{E} / \mathrm{A}$ ratio, which might suggest the early stage of cardiac diastolic dysfunction.

\section{Limitations of this study}

The main limitation of this study was that it was based on the analysis in a single center and involved a small number of women with PCOS and its subphenotypes. The definitions of oligomenorrhea and hirsutism were based on self-report. However, selfreported hirsutism and oligomenorrhea have been shown to correlate with both hormone levels and sonographic polycystic ovaries supporting their validity in the diagnosis of PCOS [31]. Other methods such as 3D based formula and $2 \mathrm{D}$ based formula for assessing LVM have some advantages over classical method including the ability to take more accurate measurements and higher reproducibility. Because of a wealth of published data and ease of use, especially in larger populations with previously demonstrated prognostic value a classical method was used. Isovolumic relaxation time and deceleration time would be helpful during assessment of diastolic function of left ventricle. Unfortunately, we did not measure these parameters. Because this is a crosssectional study, a causal relationship between PCOS phenotypes and early adverse cardiac remodeling could not be concluded. This study, however, can be considered a preliminary effort in understanding the links between PCOS and heart disease. Largescale studies are needed to understand and validate results herein.

\section{Conclusions}

This study showed that women in their twenties who especially fulfilled criteria for PCOS phenotype- 1 according to the Rotterdam criteria, presented with higher LVMI, reduced $\mathrm{E}$ wave velocity and increased A wave velocity (or a relatively decreased E/A ratio compared to healthy controls) could be suggestive of impaired relaxation pattern related with diastolic dysfunction. Early detection of asymptomatic impairment in myocardial relaxation in these patients may provide appropriate strategy for prevention of CVD. Additional large scale prospective studies with detailed PCOS phenotypic data and longer follow-up periods are needed to confirm these results.

\section{Conflict of interest: None declared}

\section{References}

1. Ehrmann DA. Polycystic ovary syndrome. N Engl J Med. 2005; 352(12): 1223-1236, doi: 10.1056/NEJMra041536, indexed in Pubmed: 15788499.

2. Rotterdam ESHRE/ASRM-Sponsored PCOS Consensus Workshop Group. Revised 2003 consensus on diagnostic criteria and long-term health risks related to polycystic ovary syndrome (PCOS). Hum Reprod. 2004; 19(1): 41-47, indexed in Pubmed: 14688154.

3. Broekmans FJ, Knauff EAH, Valkenburg O, et al. PCOS according to the Rotterdam consensus criteria: Change in prevalence among WHO-II anovulation and association with metabolic factors. BJOG. 2006; 113(10): 1210-1217, doi: 10.1111/j.14710528.2006.01008.x, indexed in Pubmed: 16972863.

4. Giallauria F, Orio F, Palomba S, et al. Cardiovascular risk in women with polycystic ovary syndrome. J Cardiovasc Med (Hagerstown). 2008; 9(10): 987-992, doi: 10.2459/ JCM.0b013e32830b58d4, indexed in Pubmed: 18799960.

5. Lo JC, Feigenbaum SL, Yang J, et al. Epidemiology and adverse cardiovascular risk profile of diagnosed polycystic ovary syndrome. J Clin Endocrinol Metab. 2006; 91(4): 1357-1363, doi: 10.1210/jc.2005-2430, indexed in Pubmed: 16434451.

6. van Santbrink EJ, Hop WC, Fauser BC. Classification of normogonadotropic infertility: polycystic ovaries diagnosed by ultrasound versus endocrine characteristics of polycystic ovary syndrome. Fertil Steril. 1997; 67(3): 452-458, indexed in Pubmed: 9091329.

7. Matthews DR, Hosker JP, Rudenski AS, et al. Homeostasis model assessment: insulin resistance and beta-cell function from fasting plasma glucose and insulin concentrations in man. Diabetologia. 1985; 28(7): 412-419, indexed in Pubmed: 3899825. 
8. Lang RM, Badano LP, Mor-Avi V, et al. Recommendations for cardiac chamber quantification by echocardiography in adults: an update from the American Society of Echocardiography and the European Association of Cardiovascular Imaging. J Am Soc Echocardiogr. 2015; 28(1): 1-39.e14, doi: 10.1016/j. echo.2014.10.003, indexed in Pubmed: 25559473.

9. Devereux RB, Alonso DR, Lutas EM, et al. Echocardiographic assessment of left ventricular hypertrophy: comparison to necropsy findings. Am J Cardiol. 1986; 57(6): 450-458, indexed in Pubmed: 2936235.

10. Lin SL, Tak T, Kawanishi DT, et al. Comparison of Doppler echocardiographic and hemodynamic indexes of left ventricular diastolic properties in coronary artery disease. Am J Cardiol. 1988; 62(13): 882-886, indexed in Pubmed: 3177234.

11. Apridonidze T, Essah PA, Iuorno MJ, et al. Prevalence and characteristics of the metabolic syndrome in women with polycystic ovary syndrome. J Clin Endocrinol Metab. 2005; 90(4): 19291935, doi: 10.1210/jc.2004-1045, indexed in Pubmed: 15623819.

12. Kelly CJG, Speirs A, Gould GW, et al. Altered vascular function in young women with polycystic ovary syndrome. J Clin Endocrinol Metab. 2002; 87(2): 742-746, doi: 10.1210/jcem.87.2.8199, indexed in Pubmed: 11836314.

13. Legro RS, Finegood D, Dunaif A. A fasting glucose to insulin ratio is a useful measure of insulin sensitivity in women with polycystic ovary syndrome. J Clin Endocrinol Metab. 1998; 83(8): 2694-2698, doi: 10.1210/jcem.83.8.5054, indexed in Pubmed: 9709933.

14. Di Bello V, Santini F, Di Cori A, et al. Obesity cardiomyopathy: is it a reality? An ultrasonic tissue characterization study. J Am Soc Echocardiogr. 2006; 19(8): 1063-1071, doi: 10.1016/j. echo.2006.03.033, indexed in Pubmed: 16880104.

15. Jialal I, Naiker P, Reddi K, et al. Evidence for insulin resistance in nonobese patients with polycystic ovarian disease. J Clin Endocrinol Metab. 1987; 64(5): 1066-1069, doi: 10.1210/jcem-64-51066, indexed in Pubmed: 2951393.

16. Wong CY, O'Moore-Sullivan T, Leano R, et al. Alterations of left ventricular myocardial characteristics associated with obesity. Circulation. 2004; 110(19): 3081-3087, doi: 10.1161/01.CIR.0000 147184.13872.0F, indexed in Pubmed: 15520317.

17. Victor VM, Rocha M, Bañuls C, et al. Induction of oxidative stress and human leukocyte/endothelial cell interactions in polycystic ovary syndrome patients with insulin resistance. J Clin Endocrinol Metab. 2011; 96(10): 3115-3122, doi: 10.1210/ jc.2011-0651, indexed in Pubmed: 21778215.

18. Di Bello V, Santini F, Di Cori A, et al. Relationship between preclinical abnormalities of global and regional left ventricular function and insulin resistance in severe obesity: a Color Doppler Imaging Study. Int J Obes (Lond). 2006; 30(6): 948-956, doi: 10.1038/sj.ijo.0803206, indexed in Pubmed: 16446750.

19. Goverde AJ, van Koert AJB, Eijkemans MJ, et al. Indicators for metabolic disturbances in anovulatory women with polycystic ovary syndrome diagnosed according to the Rotterdam consensus criteria. Hum Reprod. 2009; 24(3): 710-717, doi: 10.1093/ humrep/den433, indexed in Pubmed: 19095675.

20. Yilmaz M, Isaoglu U, Delibas IB, et al. Anthropometric, clinical and laboratory comparison of four phenotypes of polycystic ovary syndrome based on Rotterdam criteria. J Obstet Gynaecol Res. 2011; 37(8): 1020-1026, doi: 10.1111/j.1447-0756.2010.01478.x, indexed in Pubmed: 21481088.

21. Orio F, Palomba S, Spinelli L, et al. The cardiovascular risk of young women with polycystic ovary syndrome: an observational, analytical, prospective case-control study. J Clin Endocrinol Metab. 2004; 89(8): 3696-3701, doi: 10.1210/jc.2003-032049, indexed in Pubmed: 15292291.

22. Selcoki Y, Yilmaz OC, Carlioglu A, et al. Cardiac flow parameters with conventional and pulsed tissue Doppler echocardiography imaging in patients with polycystic ovary syndrome. Gynecol Endocrinol. 2010; 26(11): 815-818, doi: 10.3109/09513590.2010 .487596, indexed in Pubmed: 20486878.

23. Kosmala W, O'Moore-Sullivan TM, Plaksej R, et al. Subclinical impairment of left ventricular function in young obese women: contributions of polycystic ovary disease and insulin resistance. J Clin Endocrinol Metab. 2008; 93(10): 3748-3754, doi: 10.1210/ jc.2008-1017, indexed in Pubmed: 18682502.

24. Tíras MB, Yalcin R, Noyan V, et al. Alterations in cardiac flow parameters in patients with polycystic ovarian syndrome. Hum Reprod. 1999; 14(8): 1949-1952, indexed in Pubmed: 10438405.

25. Tekin A, Tekin G, Cölkesen Y, et al. Left ventricular function in patients with polycystic ovary syndrome: a Doppler echocardiographic study. Exp Clin Endocrinol Diabetes. 2009; 117(4): 165-169, doi: 10.1055/s-2008-1080923, indexed in Pubmed: 19085702.

26. Koren MJ, Devereux RB, Casale PN, et al. Relation of left ventricular mass and geometry to morbidity and mortality in uncomplicated essential hypertension. Ann Intern Med. 1991; 114(5): 345-352, indexed in Pubmed: 1825164.

27. Zimmermann S, Phillips RA, Dunaif A, et al. Polycystic ovary syndrome: lack of hypertension despite profound insulin resistance. J Clin Endocrinol Metab. 1992; 75(2): 508-513, doi: 10.1210/jcem.75.2.1639952, indexed in Pubmed: 1639952.

28. Wild RA, Carmina E, Diamanti-Kandarakis E, et al. Assessment of cardiovascular risk and prevention of cardiovascular disease in women with the polycystic ovary syndrome: a consensus statement by the Androgen Excess and Polycystic Ovary Syndrome (AE-PCOS) Society. J Clin Endocrinol Metab. 2010; 95(5): 2038-2049, doi: 10.1210/jc.2009-2724, indexed in Pubmed: 20375205.

29. Abhayaratna WP, Seward JB, Appleton CP, et al. Left atrial size: physiologic determinants and clinical applications. J Am Coll Cardiol. 2006; 47(12): 2357-2363, doi: 10.1016/j.jacc.2006.02.048, indexed in Pubmed: 16781359.

30. Yarali H, Yildirir A, Aybar F, et al. Diastolic dysfunction and increased serum homocysteine concentrations may contribute to increased cardiovascular risk in patients with polycystic ovary syndrome. Fertil Steril. 2001; 76(3): 511-516, indexed in Pubmed: 11532474.

31. Taponen S, Martikainen H, Järvelin MR, et al. Northern Finland Birth Cohort 1966 Study. Hormonal profile of women with selfreported symptoms of oligomenorrhea and/or hirsutism: Northern Finland birth cohort 1966 study. J Clin Endocrinol Metab. 2003; 88(1): 141-147, doi: 10.1210/jc.2002-020982, indexed in Pubmed: 12519843. 